\title{
Synthesis of New Derivatives of Heterocyclic Compounds Containing Pyridine, Pyrimidine and Triazole Ortho-Fused to Isoquinoline Moiety
}

\author{
Hamdi M. Hassaneen ${ }^{1}$, Nada M. Abunada ${ }^{2}$, Huwaida M. E. Hassaneen ${ }^{1} \&$ Omar A. Miqdad $^{2}$ \\ ${ }^{1}$ Department of Chemistry, Faculty of Science, Cairo University, Giza, Egypt \\ ${ }^{2}$ Department of Chemistry, Faculty of Applied Sciences, Al-Aqsa University, Gaza, Palestine \\ Correspondence: Hamdi M. Hassaneen, Department of Chemistry, Faculty of Science, Cairo University, Giza \\ 12613, Egypt. E-mail: hamdihass@gmail.com
}

\author{
Received: April 16, 2012 Accepted: May 3, $2012 \quad$ Online Published: May 27, 2012 \\ doi:10.5539/ijc.v4n3p86 URL: http://dx.doi.org/10.5539/ijc.v4n3p86
}

\begin{abstract}
4-Amino-9,10-dimethoxy-2-phenyl-6,7-dihydro-2(H)-pyrido[2,1-a]isoquinoline-1,3-dicarbonitrile derivatives 4a-c were obtained from the reaction of 6,7-dimethoxy-3,4-dihydroisoqinoline-1-acetonitrile $\mathbf{1}$ with arylidenemalononitrile $\mathbf{2 a - c}$ in boiling acetonitile in the presence of piperidine. The reaction of $\mathbf{4 a - c}$ with triethyl orthoformate in acetic anhydride at reflux give the ethoxymethyleneamino derivatives 5a-c. Compounds 5a-c were reacted with hydrazine hydrate and give the corresponding 4-amino-3-imino-2-aryl-3,4,8,9tetrahydro-2(H)-pyrimido $\left[5^{\prime}, 4^{\prime}: 5,6\right]$ pyrido [2,1-a]isoquinoline-1-carbonitrile derivatives 6a-c. Refluxing compound $\mathbf{6 a}$ in an excess of triethyl orthoformate give 7a. When compounds 6a-c were refluxed with acetic anhydride or benzoyl chloride in pyridine, they afford the corresponding 4-methyl and 4-phenyl derivatives 8a-c and 9a-c, respectively. Compound 6a was refluxed in diethyloxalate and give product 10. Also, the reaction of 6a with C-acylhydrazonoyl halides 13a,b in refluxing chloroform in the presence of triethylamine afford 14a,b. Correct elemental analyses and spectral data (IR, ${ }^{1} \mathrm{H}$ NMR, ${ }^{13} \mathrm{C}$ NMR and MS) confirm the structure of the synthesized compounds.
\end{abstract}

Keywords: pyrido[2,1-a]isoquinoline, triazolopyrimidopyridoiso-quinoline, triazinolopyrimidopyridoiso -quinoline

\section{Introduction}

Isoquinoline derivatives have been shown to possess a wide range of biological activities including anticancer (Mukherjee et al., 2010; Knolker \& Agarwal, 2005), anti-inflammatory (Barbosa-Filho et al., 2006) antidepressant (Maryanoff et al. 1984; Rajagopalan 1984), antimalarial (Buchana et al., 2009) and anti-HIV (Kashiwada et al., 2005). They also act as potential cetylcholinesterase inhibitors (Markmee et al., 2006), as $a_{2}$-adrenoreceptor antagonist (Chung et al., 2000) and exhibit antidepressant (Maryanoff et al., 1987) and antispasmodic effects (Chandra et al., 2001). Also, pyrimidines and pyridopyrimidines are reported to show a broad spectrum of pharmacological properties such as antimicrobial (Kanth et al., 2006; Chan et al., 2005; Vry et al., 2004), central nervous system (CNS) depressant, analgesic, anti-inflammatory (Sondhi et al., 1999; Boyle et al., 2001; Lee et al., 2001; Hafez et al., 2008), and anti-HIV (Rawal et al., 2007). In addition, $[1,2,4]$ triazolo[4,3-a]pyrimidines are pharmacological scaffold that represent a wide range of biological activities such as antitumor (Hafez \& El-Gazzar, 2009), anti-inflammatory (Hafez et al., 2008), adenosine $\mathrm{A}_{2 \mathrm{a}}$ receptor antagonist (Vu et al., 2004), acetohydroxy acid synthase inhibitor (Chen et al., 2010) and antimalaria parasite (Phillips et al., 2008). Thus, it was of interest to synthesize ring systems combining the isoquinoline, pyridine, pyrimidine and triazole moieties in order of their possible biological properties. Although several examples of the triazoloazines have been reported in literature (Elnagdi et al., 1990; Quiroga et al., 1999; Elwan et al., 1996; Hassaneen et al., 2001) there is only our previous report contains 1,2,4-triazole ring fused with pyrimidopyridoisoquinoline ring system (Abdallah et al., 2009). In continuation of our previous work on isoquinoline, the present study was designed to synthesis some new derivatives of tri-, tetra- and pentaortho-fused heterocyclic compounds especially pyridoisoquinoline, pyrimidopyridoisoquinoline and triazolo/triazinopyrimidopyridoisoquinoline. 


\section{Experimental}

General. Melting points were determined in open glass capillaries with a Gallenkamp apparatus. The infrared spectra were recorded in potassium bromide disks on a Pye Unicam SP 3-300 and Shimadzu FTIR 8101 PC infrared spectrophotometer. NMR spectra were recorded with a Varian Mercury VXR-300 NMR spectrometer at $300 \mathrm{MHz}\left({ }^{1} \mathrm{H} \mathrm{NMR}\right)$ and at $75 \mathrm{MHz}\left({ }^{13} \mathrm{C}\right.$ NMR) using $\mathrm{CDCl}_{3}$ as solvent and TMS as internal standard. Mass spectra (EI) were obtained at $70 \mathrm{eV}$ with a type Shimadzu GCMS QP 1000 EX spectrometer. Microwave irradiation was carried out using a CEM Discover Labmate ${ }^{\mathrm{TM}}$ microwave apparatus $\left(300 \mathrm{~W}\right.$ with ChemDriver ${ }^{\mathrm{TM}}$ Software). Elemental analyses were carried out at the Micro analytical Center of Cairo University. 6,7-dimethoxy-3,4-dihydroisoqinoline-1-acetonitrile 1 (Osbond, 1951) and the hydrazonoyl halides 13a (Eweiss \& Osman, 1980), 13b (Shawali \& Abdelhamid, 1976) and 15 (Shawali et al. 1975) were prepared as previously described.

\subsection{Synthesis of Compounds 4a-c}

To a solution of arylidenemalononitrile $(5 \mathrm{mmol})$ and 6,7-dimethoxy-3,4-dihydroisouinolin-1-acetonitrile $(1.15 \mathrm{~g}$, $5 \mathrm{mmol})$ in acetonitrile $(40 \mathrm{~mL})$ was added 4 drops of piperidine at room temperature. The reaction mixture was refluxed for $3 \mathrm{~h}$. The solvent was evaporated under reduced pressure and the residue was triturated with methanol $(10 \mathrm{~mL})$ where it solidified. The crude product was collected and crystallized from dimethylformamide to give 4a-c. The compounds 4a-c were also prepared by refluxing equimolar amounts of $\mathbf{1}$, the appropriate aldehyde and malononitrile under similar conditions described above.

4-Amino-2-(4-chlorophenyl)-9,10-dimethoxy-6,7-dihydro-2(H)-pyrido[2,1-a]isoquinoline-1,3-dicarbonitrile 4a. yield $86 \%$, mp $2366^{\circ} \mathrm{C}$, IR $(\mathrm{KBr}) v_{\max } 2152(\mathrm{C} \equiv \mathrm{N}), 2186(\mathrm{C} \equiv \mathrm{N}), 3354,3471\left(\mathrm{NH}_{2}\right) \mathrm{cm}^{-1} .{ }^{1} \mathrm{H} \mathrm{NMR}\left(\mathrm{CDCl}_{3}\right) \delta 2.8$ $(\mathrm{m}, 2 \mathrm{H}$, isoquinoline- $4 \mathrm{H}), 3.5\left(\mathrm{~m}, 1 \mathrm{H}\right.$, isoquinoline-3H), $3.9\left(\mathrm{~s}, 6 \mathrm{H}\right.$, two $\left.\mathrm{OCH}_{3}\right), 4.0(\mathrm{~m}, 1 \mathrm{H}$, isoquinoline- $3 \mathrm{H})$, $4.3\left(\mathrm{~s}, 2 \mathrm{H}, \mathrm{NH}_{2}\right), 4.4(\mathrm{~s}, 1 \mathrm{H}$, pyridine- $4 \mathrm{H}), 6.7(\mathrm{~s}, 1 \mathrm{H}$, isoquinoline- $8 \mathrm{H}), 7.2-7.4(\mathrm{~m}, 4 \mathrm{H}), 7.7(\mathrm{~s}, 1 \mathrm{H}$, isoquinoline-5H) ppm. Ms: m/z $420\left(\mathrm{M}^{+}+2\right), 419,418\left(\mathrm{M}^{+}\right), 351,321,307,263,176,140,75$. Anal. Calcd. For $\mathrm{C}_{23} \mathrm{H}_{19} \mathrm{ClN}_{4} \mathrm{O}_{2}$ (418.87) C, 65.94; $\mathrm{H}, 4.57 ; \mathrm{N}, 13.37$. Found $\mathrm{C}, 65.62 ; \mathrm{H}, 4.48 ; \mathrm{N}, 13.10 \%$

4-Amino-2-(1,3-benzodioxol-5-yl)-9,10-dimethoxy-6,7-dihydro-2(H)-pyrido[2,1-a]isoquinoline-1,3-dicarbonitril e 4b. yield $80 \%$, mp $137^{\circ} \mathrm{C}$, IR $(\mathrm{KBr}) v_{\max } 2179(\mathrm{C} \equiv \mathrm{N}), 2185(\mathrm{C} \equiv \mathrm{N}), 3232,3364\left(\mathrm{NH}_{2}\right) \mathrm{cm}^{-1} .{ }^{1} \mathrm{H} \mathrm{NMR}\left(\mathrm{CDCl}_{3}\right)$ $\delta 2.9(\mathrm{~m}, 2 \mathrm{H}$, isoquinoline- $4 \mathrm{H}), 3.5(\mathrm{~m}, 1 \mathrm{H}$, isoquinoline- $3 \mathrm{H}), 3.8\left(\mathrm{~s}, 3 \mathrm{H}, \mathrm{OCH}_{3}\right), 3.9\left(\mathrm{~s}, 3 \mathrm{H}, \mathrm{OCH}_{3}\right), 4.0(\mathrm{~m}, 1 \mathrm{H}$, isoquinoline- $3 \mathrm{H}), 4.2\left(\mathrm{~s}, 2 \mathrm{H}, \mathrm{NH}_{2}\right), 4.3(\mathrm{~s}, 1 \mathrm{H}$, pyridine- $4 \mathrm{H}), 6.0\left(\mathrm{~s}, 2 \mathrm{H}, \mathrm{OCH}_{2} \mathrm{O}\right), 6.7(\mathrm{~s}, 1 \mathrm{H}$, isoquinoline- $8 \mathrm{H})$, 6.8-7.3 (m, 3H), $7.7\left(\mathrm{~s}, 1 \mathrm{H}\right.$, isoquinoline-5H) ppm. MS: $\mathrm{m} / \mathrm{z} 429\left(\mathrm{M}^{+}+1\right), 428\left(\mathrm{M}^{+}\right), 427,426,381,361,307,291$, 268, 63. Anal. Calcd. For $\mathrm{C}_{24} \mathrm{H}_{20} \mathrm{~N}_{4} \mathrm{O}_{4}$ (428.14) C, 67.28; H, 4.71; N, 13.08. Found C, 67.09; H, 4.61; N, 13.00\%.

4-Amino-2-(4-methoxyphenyl)-9,10-dimethoxy-6,7-dihydro-2(H)-pyrido[2,1-a]isoquinoline-1,3-dicarbonitrile 4c. yield 77\%, mp $192^{\circ} \mathrm{C}$, IR (KBr) $v_{\max } 2187(\mathrm{C} \equiv \mathrm{N}), 2197(\mathrm{C} \equiv \mathrm{N}), 3354,3471\left(\mathrm{NH}_{2}\right) \mathrm{cm}^{-1} .{ }^{1} \mathrm{H}$ NMR (DMSO-d 6 ) $\delta 2.8(\mathrm{~m}, 2 \mathrm{H}$, isoquinoline- $4 \mathrm{H}), 3.5(\mathrm{~m}, 1 \mathrm{H}$, isoquinoline- $3 \mathrm{H}), 3.8\left(\mathrm{~s}, 3 \mathrm{H}, \mathrm{OCH}_{3}\right), 3.9\left(\mathrm{~s}, 3 \mathrm{H}, \mathrm{OCH}_{3}\right), 4.0(\mathrm{~s}, 3 \mathrm{H}$, $\left.\mathrm{OCH}_{3}\right), 4.0(\mathrm{~m}, 1 \mathrm{H}$, isoquinoline- $3 \mathrm{H}), 4.3\left(\mathrm{~s}, 2 \mathrm{H}, \mathrm{NH}_{2}\right), 4.4(\mathrm{~s}, 1 \mathrm{H}$, pyridine- $4 \mathrm{H}), 6.7(\mathrm{~s}, 1 \mathrm{H}$, isoquinoline- $8 \mathrm{H})$, 6.9-7.3 (m, 4H), $7.7\left(\mathrm{~s}, 1 \mathrm{H}\right.$, isoquinoline-5H) ppm. ${ }^{13} \mathrm{C}$ NMR (DMSO-d $\left.{ }_{6}\right) \delta 29.84,44.13,56.88,57.55,60.17$, $86.17,112.58,115.99,116.09,120.55,121.77,122.84,123.21,129.68,130.02,132.27,138.22,147.12,148.52$, 152.44, 154.45, 177.36 ppm. MS: m/z $414\left(\mathrm{M}^{+}\right), 348,317,289,275,238,169,139$, 75. Anal. Calcd. For $\mathrm{C}_{24} \mathrm{H}_{22} \mathrm{~N}_{4} \mathrm{O}_{3}(414.45) \mathrm{C}, 69.55 ; \mathrm{H}, 5.35 ; \mathrm{N}, 13.52$. Found C, 69.29; H, 5.22; N, 13.30\%.

\subsection{Synthesis of Compounds $5 a-c$}

A mixture of 4-amino-2-aryl-9,10-dimethoxy-6,7-dihydro-2(H)-pyrido[2,1-a]isoquinoline-1,3-dicarbonitrile derivatives 4 a-c $(5 \mathrm{mmol})$, triethyl orthoformate $(3 \mathrm{~mL})$ and acetic anhydride $(20 \mathrm{~mL})$ was heated under reflux for $5 \mathrm{~h}$. The excess of acetic anhydride was distilled off under reduced pressure and the solid that precipitated on cooling was filtered. The crude product was crystallized from the proper solvent to give 5a-c.

2-(4-Chlorophenyl)-9,10-dimethoxy-4-ethoxymethyleneamino-6,7-dihydro-2(H)-pyrido[2,1-a]isoquinoline-1,3-d icarbonitrile 5a. $\mathrm{mp} 205{ }^{\circ} \mathrm{C}$ (benzene), yield $84 \%$, IR $(\mathrm{KBr}) v_{\max } 2185(\mathrm{C} \equiv \mathrm{N}), 2199(\mathrm{C} \equiv \mathrm{N}) \mathrm{cm}^{-1} .{ }^{1} \mathrm{H}$ NMR $\left(\mathrm{CDCl}_{3}\right) \delta 1.4(\mathrm{t}, \mathrm{J}=7 \mathrm{~Hz}, 3 \mathrm{H}), 2.8(\mathrm{~m}, 2 \mathrm{H}), 3.5(\mathrm{~m}, 1 \mathrm{H}), 3.8(\mathrm{~m}, 1 \mathrm{H}), 3.9\left(\mathrm{~s}, 3 \mathrm{H}, \mathrm{OCH}_{3}\right), 4.0\left(\mathrm{~s}, 3 \mathrm{H}, \mathrm{OCH}_{3}\right), 4.4$ $(\mathrm{q}, \mathrm{J}=7 \mathrm{~Hz}, 2 \mathrm{H}), 4.5(\mathrm{~s}, 1 \mathrm{H}$, pyridine- $4 \mathrm{H}), 6.7(\mathrm{~s}, 1 \mathrm{H}$, isoquinoline-8H), 7.2-7.4 $(\mathrm{m}, 4 \mathrm{H}), 7.8(\mathrm{~s}, 1 \mathrm{H}$, isoquinoline-5H), $8.00(\mathrm{~s}, 1 \mathrm{H}, \mathrm{N}=\mathrm{CH}) \mathrm{ppm}$. MS: $\mathrm{m} / \mathrm{z} 476\left(\mathrm{M}^{+}+2\right), 475,474\left(\mathrm{M}^{+}\right), 417,402,335,307,291,233$, 167, 75. Anal. Calcd. For $\mathrm{C}_{26} \mathrm{H}_{23} \mathrm{ClN}_{4} \mathrm{O}_{3}$ (474.93) C, 65.74; H, 4.88; N, 11.80. Found, C, 65.55; H, 4.74; N, $11.67 \%$.

2-(1,3-Benzodioxol-5-yl)-4-ethoxymethyleneamino-9,10-dimethoxy-6,7-dihydro-2(H)-pyrido[2,1-a]isoquinoline -1,3-dicarbonitrile 5b. $\mathrm{mp} 182{ }^{\circ} \mathrm{C}$ (ethanol), yield 83\%, IR (KBr) $v_{\max } 2185(\mathrm{C} \equiv \mathrm{N}), 2197(\mathrm{C} \equiv \mathrm{N}) \mathrm{cm}^{-1} .{ }^{1} \mathrm{H} \mathrm{NMR}$ $\left(\mathrm{CDCl}_{3}\right) \delta 1.4(\mathrm{t}, \mathrm{J}=7 \mathrm{~Hz}, 3 \mathrm{H}), 2.8(\mathrm{~m}, 2 \mathrm{H}$, isoquinoline- $4 \mathrm{H}), 3.5(\mathrm{~m}, 1 \mathrm{H}$, isoquinoline- $3 \mathrm{H}), 3.8(\mathrm{~m}, 1 \mathrm{H}$, 
isoquinoline-3H), $3.9\left(\mathrm{~s}, 3 \mathrm{H}, \mathrm{OCH}_{3}\right), 4.0\left(\mathrm{~s}, 3 \mathrm{H}, \mathrm{OCH}_{3}\right), 4.2(\mathrm{~s}, 1 \mathrm{H}$, pyridine- $4 \mathrm{H}), 4.4(\mathrm{q}, \mathrm{J}=7 \mathrm{~Hz}, 2 \mathrm{H}), 6.0(\mathrm{~s}$, $\left.2 \mathrm{H}, \mathrm{OCH}_{2} \mathrm{O}\right), 6.7(\mathrm{~s}, 1 \mathrm{H}$, isoquinoline- $8 \mathrm{H}), 6.9-7.4(\mathrm{~m}, 3 \mathrm{H}), 7.8(\mathrm{~s}, 1 \mathrm{H}$, isoquinoline-5H), $8.02(\mathrm{~s}, 1 \mathrm{H}, \mathrm{N}=\mathrm{CH})$ ppm. MS: $\mathrm{m} / \mathrm{z} 486\left(\mathrm{M}^{+}+2\right), 484\left(\mathrm{M}^{+}\right), 445,412,335,307,290,247,178,126$. Anal. Calcd. $\mathrm{For}_{\mathrm{C}_{27}} \mathrm{H}_{24} \mathrm{~N}_{4} \mathrm{O}_{5}$ (484.50) C, 65.74; H, 4.88; N, 11.80. Found, C, 65.55; H, 4.74; N, 11.67\%.

2-(4-Methoxyphenyl)-4-ethoxymethyleneamino-9,10-dimethoxy-6,7-dihydro-2(H)-pyrido[2,1-a]isoquinoline-1,3 -dicarbonitrile 5c. $\mathrm{mp} 175{ }^{\circ} \mathrm{C}$ (ethanol), yield 83\%, IR (KBr) $v_{\max } 2191(\mathrm{C} \equiv \mathrm{N}), 2200(\mathrm{C} \equiv \mathrm{N}) \mathrm{cm}^{-1} \cdot{ }^{1} \mathrm{H}$ NMR $\left(\mathrm{DMSO}_{\mathrm{d}}\right) \delta 1.4(\mathrm{t}, \mathrm{J}=7 \mathrm{~Hz}, 3 \mathrm{H}), 2.8(\mathrm{~m}, 2 \mathrm{H}$, isoquinoline-4H), $3.5(\mathrm{~m}, 1 \mathrm{H}$, isoquinoline-3H), $3.8(\mathrm{~s}, 3 \mathrm{H}$, $\left.\mathrm{OCH}_{3}\right), 3.8(\mathrm{~m}, 1 \mathrm{H}$, isoquinoline- $3 \mathrm{H}), 3.9\left(\mathrm{~s}, 3 \mathrm{H}, \mathrm{OCH}_{3}\right), 4.0\left(\mathrm{~s}, 3 \mathrm{H}, \mathrm{OCH}_{3}\right), 4.4(\mathrm{q}, \mathrm{J} 7 \mathrm{~Hz}, 2 \mathrm{H}), 4.5(\mathrm{~s}, 1 \mathrm{H}$, pyridine-4H), $6.7(\mathrm{~s}, 1 \mathrm{H}$, isoquinoline- $8 \mathrm{H}), 6.9-7.3(\mathrm{~m}, 4 \mathrm{H}), 7.8(\mathrm{~s}, 1 \mathrm{H}$, isoquinoline-5H), $8.0(\mathrm{~s}, 1 \mathrm{H}, \mathrm{N}=\mathrm{CH})$ ppm. ${ }^{13} \mathrm{C}$ NMR (DMSO-d 6 ) $\delta 15.73,30.04,44.04,56.90,57.46,57.55,65.63,73.79,83.61,112.81,115.72$, 116.07, 120.64, 121.58, 122.97, 130.07, 130.49, 133.51, 137.86, 146.45, 148.45, 152.73, 155.01, 160.67, 162.51 ppm. MS: m/z $470\left(\mathrm{M}^{+}\right), 412,398,367,335,305,291,233,167$, 75. Anal. Calcd. For $\mathrm{C}_{27} \mathrm{H}_{26} \mathrm{~N}_{4} \mathrm{O}_{4}(470.52) \mathrm{C}$, 68.92; H, 5.57; N, 11.91. Found C, 68.82; H, 5.45; N, 11.76\%

\subsection{Synthesis of Compounds $6 a-c$}

Hydrazine hydrate $(10 \mathrm{~mL})$ was added to a suspension of $\mathbf{5 a - c}(10 \mathrm{mmol})$ in ethanol $(40 \mathrm{~mL})$. The reaction mixture was stirred at room temperature for $4 \mathrm{~h}$. The precipitate which formed was filtered off, washed with water, dried in air and crystallized from the indicated solvent to give product 6a-c.

4-Amino-2-(chlorophenyl)-11,12-dimethoxy-3-imino-3,4,8,9-tetrahydro-2(H)-pyrimido[5',4':5,6]pyrido[2,1-a]is oquinoline-1-carbonitrile 6a. $\mathrm{mp} 180{ }^{\circ} \mathrm{C}$ (acetic acid), yield 92\%, IR (KBr) $v_{\max } 2200(\mathrm{C} \equiv \mathrm{N}), 3160(\mathrm{NH}), 3301$, $3321\left(\mathrm{NH}_{2}\right) \mathrm{cm}^{-1} .{ }^{1} \mathrm{H}$ NMR $\left(\mathrm{CDCl}_{3}\right) \delta 2.8(\mathrm{~m}, 2 \mathrm{H}$, isoquinoline-4H), $3.7(\mathrm{~m}, 1 \mathrm{H}$, isoquinoline- $3 \mathrm{H}), 3.8(\mathrm{~s}, 3 \mathrm{H}$, $\left.\mathrm{OCH}_{3}\right), 3.9\left(\mathrm{~s}, 3 \mathrm{H}, \mathrm{OCH}_{3}\right), 4.5\left(\mathrm{~m}, 1 \mathrm{H}\right.$, isoquinoline-3H), $4.6(\mathrm{~s}, 1 \mathrm{H}$, pyridine- $4 \mathrm{H}), 4.8\left(\mathrm{~s}, 2 \mathrm{H}, \mathrm{NH}_{2}\right), 5.90(\mathrm{~s}, 1 \mathrm{H}$, $\mathrm{NH}), 6.7(\mathrm{~s}, 1 \mathrm{H}$, isoquinoline-8H), 7.2-7.4 (m, 4H, ArH's), $7.7(\mathrm{~s}, 1 \mathrm{H}$, isoquinoline-5H), $8.66(\mathrm{~s}, 1 \mathrm{H}$, pyrimidine-2H) ppm. ${ }^{13} \mathrm{C}$ NMR $\left(\mathrm{CDCl}_{3}\right) \delta 28.48,45.21,55.35,55.60,78.89,97.19,110.93,111.32,119.93$, $120.01,122.00,128.30,130.19,131.61,133.01,145.01,145.38,145.93,146.50,149.63,150.02,153.92$. MS: $\mathrm{m} / \mathrm{z} 462,460,445,428,339,324,308,270,182,166,140,120,91,65$. Anal. Calcd. For $\mathrm{C}_{24} \mathrm{H}_{21} \mathrm{ClN}_{6} \mathrm{O}_{2}(460.91)$ C, 62.53; H, 4.59; N, 18.23. Found C, 62.45; H, 4.46; N, 18.20\%.

4-Amino-2-(1,3-benzodioxol-5-yl)-11,12-dimethoxy-3-imino-3,4,8,9-tetrahydro-2(H)-pyrimido[5',4':5,6]pyrido[ 2,1-a]isoquinoline-1-carbonitrile 6b. $\mathrm{mp} 221^{\circ} \mathrm{C}$ (acetic acid), yield 94\%, IR (KBr) $v_{\max } 2181(\mathrm{C} \equiv \mathrm{N}), 3135(\mathrm{NH})$, $3313,3438\left(\mathrm{NH}_{2}\right) \mathrm{cm}^{-1} .{ }^{1} \mathrm{H}$ NMR $\left(\mathrm{CDCl}_{3}\right) \delta 2.8(\mathrm{~m}, 2 \mathrm{H}$, isoquinoline- $4 \mathrm{H}), 3.7(\mathrm{~m}, 1 \mathrm{H}$, isoquinoline- $3 \mathrm{H}), 3.8(\mathrm{~s}$, $\left.3 \mathrm{H}, \mathrm{OCH}_{3}\right), 3.9\left(\mathrm{~s}, 3 \mathrm{H}, \mathrm{OCH}_{3}\right), 4.4(\mathrm{~m}, 1 \mathrm{H}$, isoquinoline- $3 \mathrm{H}), 4.5(\mathrm{~s}, 1 \mathrm{H}$, pyridine- $4 \mathrm{H}), 4.77\left(\mathrm{~s}, 2 \mathrm{H}, \mathrm{NH}_{2}\right), 5.87(\mathrm{~s}$, $1 \mathrm{H}, \mathrm{NH}), 5.9\left(\mathrm{~s}, 2 \mathrm{H}, \mathrm{OCH}_{2} \mathrm{O}\right), 6.7(\mathrm{~s}, 1 \mathrm{H}$, isoquinoline- $8 \mathrm{H}), 6.8-7.0(\mathrm{~m}, 3 \mathrm{H}, \mathrm{ArH}$ 's), $7.7(\mathrm{~s}, 1 \mathrm{H}$, isoquinoline- $5 \mathrm{H})$, $8.64(\mathrm{~s}, 1 \mathrm{H}$, pyrimidine- $2 \mathrm{H}) \mathrm{ppm} .{ }^{13} \mathrm{C}$ NMR $\left(\mathrm{DMSO}_{\mathrm{d}}\right) \delta$ 28.53, 44.78, 55.34, 56.08, 78.81, 97.20, 101.69, $108.38,110.41,110.94,111.31,120.03,120.18,121.03,121.21,131.66,140.96,145.42,145.87,146.43,149.92$, 150.08, 152.40, 154.74, 158.68. MS: $\mathrm{m} / \mathrm{z} 472\left(\mathrm{M}^{+}+2\right), 471,470\left(\mathrm{M}^{+}\right), 455,438,349,334,318,260,204,165$, 121, 91, 65. Anal. Calcd. For $\mathrm{C}_{25} \mathrm{H}_{22} \mathrm{~N}_{6} \mathrm{O}_{4}$ (470.48) C, 63.82; H, 4.71; N, 17.86. Found C, 63.55; H, 4.59; N, $17.75 \%$.

4-Amino-11,12-dimethoxy-3-imino-2-(methoxyphenyl)-3,4,8,9-tetrahydro-2(H)-pyrimido[5',4':5,6]pyrido[2,1-a] isoquinoline-1-carbonitrile 6 c. $\mathrm{mp} 175^{\circ} \mathrm{C}$ (acetic acid), yield 95\%, IR $(\mathrm{KBr}) v_{\max } 2179(\mathrm{C} \equiv \mathrm{N}), 3280(\mathrm{NH}), 3318$, $3435\left(\mathrm{NH}_{2}\right) \mathrm{cm}^{-1} .{ }^{1} \mathrm{H}$ NMR $\left(\mathrm{CDCl}_{3}\right) \delta 2.8(\mathrm{~m}, 2 \mathrm{H}$, isoquinoline- $4 \mathrm{H}), 3.7(\mathrm{~m}, 1 \mathrm{H}$, isoquinoline- $3 \mathrm{H}), 3.8(\mathrm{~s}, 3 \mathrm{H}$, $\left.\mathrm{OCH}_{3}\right), 3.9\left(\mathrm{~s}, 3 \mathrm{H}, \mathrm{OCH}_{3}\right), 4.0\left(\mathrm{~s}, 3 \mathrm{H}, \mathrm{OCH}_{3}\right), 4.4(\mathrm{~m}, 1 \mathrm{H}$, isoquinoline- $3 \mathrm{H}), 4.5(\mathrm{~s}, 1 \mathrm{H}$, pyridine- $4 \mathrm{H}), 4.75(\mathrm{~s}$, $\left.2 \mathrm{H}, \mathrm{NH}_{2}\right), 5.86(\mathrm{~s}, 1 \mathrm{H}, \mathrm{NH}), 6.7(\mathrm{~s}, 1 \mathrm{H}$, isoquinoline- $8 \mathrm{H}), 6.8-7.0(\mathrm{~m}, 4 \mathrm{H}), 7.7(\mathrm{~s}, 1 \mathrm{H}$, isoquinoline- $5 \mathrm{H}), 8.64(\mathrm{~s}$, $1 \mathrm{H}$, pyrimidine-2H). ${ }^{13} \mathrm{C}$ NMR $\left(\mathrm{DMSO}_{-} \mathrm{d}_{6}\right) \delta 28.47,45.03,55.98,56.01,60.09,78.81,97.82,110.97,111.16$, $116.13,120.01,120.12,122.91,129.38,131.02,131.71,145.64,146.00,146.69,149.73,150.11,153.60,154.64$. MS: $\mathrm{m} / \mathrm{z} 458,456,441,425,333,319,303,271,233,204,165,121,91,65$. Anal. Calcd. For $\mathrm{C}_{25} \mathrm{H}_{24} \mathrm{~N}_{6} \mathrm{O}_{3}$ (456.49) C, 65.77; H, 5.30; N, 18.41. Found C, 65.49; H, 5.19; N, 18.34\%.

2.4 Synthesis of 2-aryl-13,14-dimethoxy-10,11-dihydro-2(H)-1,2,4-triazolo[3",2":6',1']pyrimido[5',4':5,6]pyrido [2,1-a] isoquinoline-1-carbonitrile derivatives $7 a$

A mixture of 4-amino-2-(4-chloropheyl)-11,12-dimethoxy-3-imino-3,4,8,9-tetrahydro-2(H)-pyrimido[5',4':5,6] pyrido[2,1-a]isoquinoline-1-carbonitrile $\mathbf{6 a}(5 \mathrm{mmol})$ with triethyl orthoformate $(15 \mathrm{~mL})$ or formic acid $(15 \mathrm{~mL})$ was refluxed for $4 \mathrm{~h}$. After cooling, the precipitated product was collected by filtration and crystallized from dimethylformamide to give product 7a. $\mathrm{mp} 229^{\circ} \mathrm{C}$, yield 78\%, IR $(\mathrm{KBr}) v_{\max } 2179(\mathrm{C} \equiv \mathrm{N}) \mathrm{cm}^{-1} .{ }^{1} \mathrm{H} \mathrm{NMR}\left(\mathrm{CDCl}_{3}\right)$ $\delta 3.0(\mathrm{~m}, 2 \mathrm{H}$, isoquinoline- $4 \mathrm{H}), 3.9\left(\mathrm{~s}, 3 \mathrm{H}, \mathrm{OCH}_{3}\right), 4.0\left(\mathrm{~s}, 3 \mathrm{H}, \mathrm{OCH}_{3}\right), 4.1(\mathrm{~m}, 1 \mathrm{H}$, isoquinoline- $3 \mathrm{H}), 4.5(\mathrm{~m}, 1 \mathrm{H}$, isoquinoline-3H), $5.5(\mathrm{~s}, 1 \mathrm{H}$, pyridine- $4 \mathrm{H}), 6.8(\mathrm{~s}, 1 \mathrm{H}$, isoquinoline- $8 \mathrm{H}), 7.2-7.5(\mathrm{~m}, 4 \mathrm{H}), 7.9(\mathrm{~s}, 1 \mathrm{H}$, isoquinoline-5H), $8.32(\mathrm{~s}, 1 \mathrm{H}$, pyrimidine- $2 \mathrm{H}), 9.13\left(\mathrm{~s}, 1 \mathrm{H}\right.$, triazole-3H) ppm. ${ }^{13} \mathrm{C} \mathrm{NMR}\left(\mathrm{CDCl}_{3}\right) \delta 28.39,45.63$, 
$55.36,55.51,78.91,98.34,110.82,11.21,120.02,120.61,122.60,128.29,130.21,131.62,134.10,145.62$, 146.10, 146.71, 146.32, 149.52, 150.82, 151.72, 160.10. MS: m/z $472\left(\mathrm{M}^{+}+2\right), 471,470\left(\mathrm{M}^{+}\right), 359,285,235$, 179, 111, 75. Anal. Calcd. For $\mathrm{C}_{25} \mathrm{H}_{19} \mathrm{ClN}_{6} \mathrm{O}_{2}$ (470.91) C, 63.75; H, 4.07; N, 17.85. Found C, 63.65; H, 3.91; N, $17.60 \%$.

\subsection{Synthesis of compounds $8 a-c$}

A solution of each of $6 \mathbf{a}-\mathbf{c}(5 \mathrm{mmol})$ in acetic anhydride $(20 \mathrm{~mL})$ was refluxed for $3 \mathrm{~h}$. The reaction mixture was cooled and the solid that separated was collected and crystallized from dimethylformamide to give 8a-c.

2-(4-Chlorophenyl)-13,14-dimethoxy-4-methyl-10,11-dihydro-2(H)-1,2,4-triazolo[3", 2" :6', 1']pyrimido[5',4':5,6] pyrido[2,1-a]isoquinoline-1-carbonitrile 8a. mp $250{ }^{\circ} \mathrm{C}$, yield 74\%, IR (KBr) $v_{\max } 2182(\mathrm{C} \equiv \mathrm{N}) \mathrm{cm}^{-1} .{ }^{1} \mathrm{H} \mathrm{NMR}$ $\left(\mathrm{CDCl}_{3}\right) \delta 2.45\left(\mathrm{~s}, 3 \mathrm{H}, \mathrm{CH}_{3}\right), 2.9\left(\mathrm{~m}, 2 \mathrm{H}\right.$, isoquinoline-4H), $3.9\left(\mathrm{~m}, 1 \mathrm{H}\right.$, isoquinoline-3H), $4.0\left(\mathrm{~s}, 3 \mathrm{H}, \mathrm{OCH}_{3}\right), 4.1$ $\left(\mathrm{s}, 3 \mathrm{H}, \mathrm{OCH}_{3}\right), 4.4(\mathrm{~m}, 1 \mathrm{H}$, isoquinoline- $3 \mathrm{H}), 5.4(\mathrm{~s}, 1 \mathrm{H}$, pyridine- $4 \mathrm{H}), 6.7(\mathrm{~s}, 1 \mathrm{H}$, isoquinoline $-8 \mathrm{H}), 7.2-7.4(\mathrm{~m}$, $4 \mathrm{H}), 7.8\left(\mathrm{~s}, 1 \mathrm{H}\right.$, isoquinoline-5H), $8.99\left(\mathrm{~s}, 1 \mathrm{H}\right.$, pyrimidine-2H) ppm. ${ }^{13} \mathrm{C}$ NMR $\left(\mathrm{DMSO}-\mathrm{d}_{6}\right) \delta 14.30,28.41,45.40$, 55.62 , 55.71, 79.76, 97.30, 111.21, 119.70, 121.30, 122.00, 122.80, 132.00, 132.90, 139.00, 139.90, 144.11, 144.24, 145.62, 146.61, 147.15, 151.21, 152.30, 165.20. MS: m/z $486\left(\mathrm{M}^{+}+2\right), 485,484\left(\mathrm{M}^{+}\right), 410,373,329$, 245, 111, 75. Anal. Calcd. For $\mathrm{C}_{26} \mathrm{H}_{21} \mathrm{ClN}_{6} \mathrm{O}_{2}$ (484.93) C, 64.39; H, 4.36; N, 17.33. Found C, 64.22; H, 4.21; N, $17.20 \%$.

2-(1,3-Benzodioxol-5-yl)-13,14-dimethoxy-4-methyl-10,11-dihydro-2(H)-1,2,4-triazolo[3",2":6',1']pyrimido[5', $\left.4^{\prime}: 5,6\right]$ pyrido[2,1-a]isoquinoline-1-carbonitrile 8b. mp $221{ }^{\circ} \mathrm{C}$, yield $73 \%$, IR $(\mathrm{KBr}) v_{\max } 2184(\mathrm{C} \equiv \mathrm{N}) \mathrm{cm}^{-1} .{ }^{1} \mathrm{H}$ NMR $\left(\mathrm{CDCl}_{3}\right) \delta 2.48\left(\mathrm{~s}, 3 \mathrm{H}, \mathrm{CH}_{3}\right), 2.88(\mathrm{~m}, 2 \mathrm{H}$, isoquinoline-4H), $3.9(\mathrm{~m}, 1 \mathrm{H}$, isoquinoline-3H), $4.0(\mathrm{~s}, 3 \mathrm{H}$, $\left.\mathrm{OCH}_{3}\right), 4.1\left(\mathrm{~s}, 3 \mathrm{H}, \mathrm{OCH}_{3}\right), 4.34\left(\mathrm{~m}, 1 \mathrm{H}\right.$, isoquinoline-3H), $5.44(\mathrm{~s}, 1 \mathrm{H}$, pyridine- $4 \mathrm{H}), 5.91\left(\mathrm{~s}, 2 \mathrm{H}, \mathrm{OCH}_{2} \mathrm{O}\right), 6.7$ $\left(\mathrm{s}, 1 \mathrm{H}\right.$, isoquinoline-8H), 7.2-7.4 $(\mathrm{m}, 3 \mathrm{H}), 7.75\left(\mathrm{~s}, 1 \mathrm{H}\right.$, isoquinoline-5H), $8.96\left(\mathrm{~s}, 1 \mathrm{H}\right.$, pyrimidine-2H) $\mathrm{ppm} .{ }^{13} \mathrm{C}$ NMR $\left(\right.$ DMSO-d $\left._{6}\right) \delta 14.31,28.26,46.21,55.56,55.74,78.99,98.61,101.73,107.92,110.63,110.92,118.82$, 120.92, 121.30, 122.31, 131.90, 138.90, 142.01, 145.21, 145.36, 146.34, 146.82, 151.62, 152.36, 154.30, 157.38, 164.90. MS: m/z $496\left(\mathrm{M}^{+}+2\right), 495,494\left(\mathrm{M}^{+}\right), 468,373,357,299,219,121,65$. Anal. Calcd. For $\mathrm{C}_{27} \mathrm{H}_{22} \mathrm{~N}_{6} \mathrm{O}_{4}$ (494.50) C, 65.58; H, 4.48; N, 17.00. Found C, 65.33; H, 4.41; N, 17.01\%.

13,14-Dimethoxy-2-(4-methoxyphenyl)-4-methyl-10,11-dihydro-2(H)-1,2,4-triazolo[3" $\left.2^{\prime \prime}: 6^{\prime}, 1^{\prime}\right]$ pyrimido[5',4':5, 6]pyrido[2,1-a]isoquinoline-1-carbonitrile 8c. mp $227^{\circ} \mathrm{C}$, yield 71\%, IR (KBr) $v_{\max } 2189 \mathrm{~cm}^{-1}$. ${ }^{1} \mathrm{H} \mathrm{NMR}\left(\mathrm{CDCl}_{3}\right)$ $\delta 2.48\left(\mathrm{~s}, 3 \mathrm{H}, \mathrm{CH}_{3}\right), 2.88(\mathrm{~m}, 2 \mathrm{H}$, isoquinoline- $4 \mathrm{H}), 3.82(\mathrm{~m}, 1 \mathrm{H}$, isoquinoline- $3 \mathrm{H}), 3.93\left(\mathrm{~s}, 3 \mathrm{H}, \mathrm{OCH}_{3}\right), 4.0(\mathrm{~s}$, $\left.3 \mathrm{H}, \mathrm{OCH}_{3}\right), 4.1\left(\mathrm{~s}, 3 \mathrm{H}, \mathrm{OCH}_{3}\right), 4.34(\mathrm{~m}, 1 \mathrm{H}$, isoquinoline-3H), $5.44(\mathrm{~s}, 1 \mathrm{H}$, pyridine- $4 \mathrm{H}), 6.7(\mathrm{~s}, 1 \mathrm{H}$, isoquinoline-8H), 7.2-7.4 (m, 4H), $7.75\left(\mathrm{~s}, 1 \mathrm{H}\right.$, isoquinoline-5H), $8.98\left(\mathrm{~s}, 1 \mathrm{H}\right.$, pyrimidine-2H) ppm. ${ }^{13} \mathrm{C} \mathrm{NMR}$ $\left(\right.$ DMSO-d $\left._{6}\right) \delta 14.26,28.41,43.25,55.49,55.81,57.63,78.63,79.31,111.03,116.12,119.17,120.18,123.10$, $130.36,131.71,133.49,137.90,144.93,145.46,146.32,146.40,152.00,152.54,156.21,166.10 . \mathrm{MS}: \mathrm{m} / \mathrm{z} 482$ $\left(\mathrm{M}^{+}+2\right), 481,480\left(\mathrm{M}^{+}\right), 440,373,357,299,240,224,121,77$. Anal. Calcd. For $\mathrm{C}_{27} \mathrm{H}_{24} \mathrm{~N}_{6} \mathrm{O}_{3}(480.51) \mathrm{C}, 67.49$; H, 5.03; N, 17.49. Found C, 67.23; H, 4.90; N, 16.99\%.

\subsection{Synthesis of Compounds $9 a-c$}

To a solution of each 6a-c $(5 \mathrm{mmol})$ in pyridine $(10 \mathrm{~mL})$ benzoyl chloride $(0.7 \mathrm{~mL}, 5 \mathrm{mmol})$ was added. The reaction mixture was refluxed for $4 \mathrm{~h}$, then cooled and poured into cold hydrochloric acid $(10 \mathrm{~mL}, 10 \%)$ with stirring. The solid that precipitated was collected, washed with cold water and finally crystallized from dimethylformamide to give 9a-c.

2-(4-Chlorophenyl)-13,14-dimethoxy-4-phenyl-10,11-dihydro-2(H)-1,2,4-triazolo[3", 2"':6',1']pyrimido[5',4':5,6] pyrido[2,1-a]isoquinoline-1-carbonitrile 9a. mp $320{ }^{\circ} \mathrm{C}$, yield $68 \%$, IR (KBr) $v_{\max } 2180(\mathrm{C} \equiv \mathrm{N}) \mathrm{cm}^{-1} .{ }^{1} \mathrm{H} ~ \mathrm{NMR}$ $\left(\mathrm{CDCl}_{3}\right) \delta 2.94\left(\mathrm{~m}, 2 \mathrm{H}\right.$, isoquinoline-4H), $3.93\left(\mathrm{~m}, 1 \mathrm{H}\right.$, isoquinoline-3H), $4.05\left(\mathrm{~s}, 3 \mathrm{H}, \mathrm{OCH}_{3}\right), 4.16(\mathrm{~s}, 3 \mathrm{H}$, $\left.\mathrm{OCH}_{3}\right), 4.41(\mathrm{~m}, 1 \mathrm{H}$, isoquinoline-3H), $5.43(\mathrm{~s}, 1 \mathrm{H}$, pyridine- $4 \mathrm{H}), 6.76(\mathrm{~s}, 1 \mathrm{H}$, isoquinoline-8H), 7.21-7.94 $(\mathrm{m}$, $9 \mathrm{H}, \mathrm{Ar} ' \mathrm{H}), 7.80\left(\mathrm{~s}, 1 \mathrm{H}\right.$, isoquinoline-5H), $8.99\left(\mathrm{~s}, 1 \mathrm{H}\right.$, pyrimidine-2H) ppm. ${ }^{13} \mathrm{C}$ NMR $\left(\mathrm{DMSO}-\mathrm{d}_{6}\right) \delta 28.32$, 44.92, 55.58, 55.81, 78.69, 97.41, 111.34, 118.93, 120.83, 123.10, 127.33, 127.79, 128.69, 129.21, 131.79, 132.21, 132.63, 138.71, 139.28, 143.92, 145.13, 145.72, 145.90, 147.00, 151.45, 153.41, 164.92. MS: m/z 548 $\left(\mathrm{M}^{+}+2\right), 547,546\left(\mathrm{M}^{+}\right), 472,469,407,361,244,111$, 75. Anal. Calcd. For $\mathrm{C}_{31} \mathrm{H}_{23} \mathrm{ClN}_{6} \mathrm{O}_{2}(547.00) \mathrm{C}, 68.06 ; \mathrm{H}$, $4.24 ; \mathrm{N}, 15.36$. Found C, 67.90; H, 4.13; N, 15.23\%.

2-(1,3-Benzodioxol-5-yl)-13,14-dimethoxy-4-phenyl-10,11-dihydro-2(H)-1,2,4-triazolo[3",2":6',1']pyrimido[5', $\left.4^{\prime}: 5,6\right]$ pyrido[2,1-a]isoquinoline-1-carbonitrile 9b. mp $176{ }^{\circ} \mathrm{C}$, yield $79 \%$, IR $(\mathrm{KBr}) v_{\max } 2179(\mathrm{C} \equiv \mathrm{N}) \mathrm{cm}^{-1} .{ }^{1} \mathrm{H}$ $\operatorname{NMR}\left(\mathrm{CDCl}_{3}\right) \delta 2.90\left(\mathrm{~m}, 2 \mathrm{H}\right.$, isoquinoline-4H), $3.93\left(\mathrm{~m}, 1 \mathrm{H}\right.$, isoquinoline-3H), $4.02\left(\mathrm{~s}, 3 \mathrm{H}, \mathrm{OCH}_{3}\right), 4.14(\mathrm{~s}, 3 \mathrm{H}$, $\left.\mathrm{OCH}_{3}\right), 4.33\left(\mathrm{~m}, 1 \mathrm{H}\right.$, isoquinoline-3H), $5.44(\mathrm{~s}, 1 \mathrm{H}$, pyridine $-4 \mathrm{H}), 5.93\left(\mathrm{~s}, 2 \mathrm{H}, \mathrm{OCH}_{2} \mathrm{O}\right), 6.77(\mathrm{~s}, 1 \mathrm{H}$, isoquinoline-8H), 7.2-7.4 (m, 8H, Ar'H), $7.77\left(\mathrm{~s}, 1 \mathrm{H}\right.$, isoquinoline-5H), $8.92\left(\mathrm{~s}, 1 \mathrm{H}\right.$, pyrimidine-2H) $\mathrm{ppm} .{ }^{13} \mathrm{C}$ 
NMR (DMSO-d $\left.{ }_{6}\right) \delta 28.24,45.87,55.71,55.84,78.89,97.93,101.52,108.93,109.90,111.23,119.62,120.89$, 122.32, 123.00, 127.26, 128.63, 129.09, 131.67, 132.01, 139.74, 143.00, 145.32, 146.00, 147.14, 147.22, 150.96, 153.43, 153.92, 157.61, 168.21. MS: m/z 558, 557, 556, 530, 469, 435, 419, 361, 278, 204, 121, 65. Anal. Calcd. For $\mathrm{C}_{32} \mathrm{H}_{24} \mathrm{~N}_{6} \mathrm{O}_{4}(556.57) \mathrm{C}, 69.05 ; \mathrm{H}, 4.31 ; \mathrm{N}, 15.10$. Found $\mathrm{C}, 68.90 ; \mathrm{H}, 4.32 ; \mathrm{N}, 15.01 \%$.

13,14-Dimethoxy-2-(4-methoxyphenyl)-4-phenyl-10,11-dihydro-2(H)-1,2,4-triazolo[3", $\left.2^{\prime \prime}: 6^{\prime}, 1^{\prime}\right]$ pyrimido[5',4':5, 6]pyrido[2,1-a] isoquinoline-1-carbonitrile 9c. $\mathrm{mp} 310^{\circ} \mathrm{C}$, yield 79\%, IR $(\mathrm{KBr}) v_{\max } 2190(\mathrm{C} \equiv \mathrm{N}) \mathrm{cm}^{-1} .{ }^{1} \mathrm{H}$ NMR $\left(\mathrm{CDCl}_{3}\right) \delta 2.91\left(\mathrm{~m}, 2 \mathrm{H}\right.$, isoquinoline-4H), $3.83\left(\mathrm{~m}, 1 \mathrm{H}\right.$, isoquinoline-3H), $3.90\left(\mathrm{~s}, 3 \mathrm{H}, \mathrm{OCH}_{3}\right), 4.03(\mathrm{~s}, 3 \mathrm{H}$, $\left.\mathrm{OCH}_{3}\right), 4.12\left(\mathrm{~s}, 3 \mathrm{H}, \mathrm{OCH}_{3}\right), 4.32(\mathrm{~m}, 1 \mathrm{H}$, isoquinoline- $3 \mathrm{H}), 5.44(\mathrm{~s}, 1 \mathrm{H}$, pyridine- $4 \mathrm{H}), 6.75(\mathrm{~s}, 1 \mathrm{H}$, isoquinoline-8H), 7.18-7.90 $(\mathrm{m}, 9 \mathrm{H}), 7.75(\mathrm{~s}, 1 \mathrm{H}$, isoquinoline- $5 \mathrm{H}), 8.91(\mathrm{~s}, 1 \mathrm{H}$, pyrimidine- $2 \mathrm{H}) \mathrm{ppm} .{ }^{13} \mathrm{C}$ NMR $\left(\right.$ DMSO-d $\left._{6}\right) \delta 28.42,44.60,55.36,55.74,57.58,78.53,79.26,110.67,115.98,118.78,121.02,122.98,127.31$, $128.71,129.10,130.46,131.80,132.21,133.48,137.60,145.24,145.32,146.21,146.43,152.26,152.64,156.37$, 165.93. MS: m/z 543, 542, 456, 435, 419, 349, 333, 271, 206, 121, 77. Anal. Calcd. For $\mathrm{C}_{32} \mathrm{H}_{26} \mathrm{~N}_{6} \mathrm{O}_{3}(542.58) \mathrm{C}$, $70.82 ; \mathrm{H}, 4.83 ; \mathrm{N}, 15.50$. Found C, 70.78; H, 4.71; N, 15.33\%.

2.7 Synthesis of ethyl 2-(chlorophenyl)-1-cyano-10,11-dihydro-13,14-dimethoxy-2(H)-1,2,4- triazolo[3",2":6',1'] pyrimido [5',4':5,6]pyrido[2,1-a]isoquinoline-4-carboxylate 10

To a solution of $6 \mathrm{a}(2.30 \mathrm{~g}, 5 \mathrm{mmol})$ in ethanol $(30 \mathrm{~mL})$, diethyloxalate $(0.73 \mathrm{~g}, 5 \mathrm{mmol})$ was added. The reaction mixture was refluxed for $3 \mathrm{~h}$, then cooled and the product that separated was collected and crystallized from acetic acid to give product $10 . \mathrm{mp} 250{ }^{\circ} \mathrm{C}$, yield $74 \%$, IR $(\mathrm{KBr}) v_{\max } 1647(\mathrm{CO}), 2182(\mathrm{C} \equiv \mathrm{N}) \mathrm{cm}^{-1} .{ }^{1} \mathrm{H} \mathrm{NMR}$ $\left(\mathrm{CDCl}_{3}\right) \delta 1.4(\mathrm{t}, \mathrm{J}=7 \mathrm{~Hz}, 3 \mathrm{H}), 3.0\left(\mathrm{~m}, 2 \mathrm{H}\right.$, isoquinoline-4H), $3.9\left(\mathrm{~s}, 6 \mathrm{H}\right.$, two $\left.\mathrm{OCH}_{3}\right), 4.1(\mathrm{~m} 1 \mathrm{H}$, isoquinoline- $3 \mathrm{H}), 4.4(\mathrm{~m}, 1 \mathrm{H}$, isoquinoline- $3 \mathrm{H}), 4.5(\mathrm{q}, \mathrm{J}=7 \mathrm{~Hz}, 2 \mathrm{H}), 5.6(\mathrm{~s}, 1 \mathrm{H}$, pyridine- $4 \mathrm{H}), 6.8(\mathrm{~s}, 1 \mathrm{H}$, isoquinoline-8H), 7.2-7.5 $(\mathrm{m}, 4 \mathrm{H}), 7.9(\mathrm{~s}, 1 \mathrm{H}$, isoquinoline- $5 \mathrm{H}), 9.18(\mathrm{~s}, 1 \mathrm{H}$, pyrimidine- $2 \mathrm{H}) \mathrm{ppm}$. MS: $\mathrm{m} / \mathrm{z} 544$ $\left(\mathrm{M}^{+}+2\right), 543,542\left(\mathrm{M}^{+}\right), 513,431,403,307,263,235,113,111,75$. Anal. Calcd. For $\mathrm{C}_{28} \mathrm{H}_{23} \mathrm{ClN}_{6} \mathrm{O}_{4}(542.97) \mathrm{C}$, $61.93 ; \mathrm{H}, 4.27 ; \mathrm{N}, 15.48$. Found C, 61.05; H, 3.81; N, 14.88\%.

\subsection{Hydrolysis of Compound 10}

A suspension of $10(2.7 \mathrm{~g}, 5 \mathrm{mmol})$ in an aqueous solution of potassium hydroxide $(10 \mathrm{~mL}, 10 \%)$ was refluxed for $2 \mathrm{~h}$. The reaction mixture was cooled, poured onto hydrochloric acid $(100,1 \mathrm{~N})$. The crude product was collected and crystallized from dimethylformamide to give compound $12 . \mathrm{mp} 310{ }^{\circ} \mathrm{C}$, yield $75 \%$, IR $(\mathrm{KBr}) v_{\max }$ $1730(\mathrm{CO}), 2182(\mathrm{CN}), 3430(\mathrm{OH}) \mathrm{cm}^{-1} .{ }^{1} \mathrm{H}$ NMR $\left(\mathrm{CDCl}_{3}\right) \delta 3.0(\mathrm{~m}, 2 \mathrm{H}), 3.91\left(\mathrm{~s}, 6 \mathrm{H}\right.$, two $\left.\mathrm{OCH}_{3}\right), 4.1(\mathrm{~m}, 1 \mathrm{H})$, $4.4(\mathrm{~m}, 1 \mathrm{H}), 5.6(\mathrm{~s}, 1 \mathrm{H}), 6.8(\mathrm{~s}, 1 \mathrm{H}), 7.2-7.5(\mathrm{~m}, 4 \mathrm{H}), 7.9(\mathrm{~s}, 1 \mathrm{H}), 9.2(\mathrm{~s}, 1 \mathrm{H}), 12.5(\mathrm{~s}, 1 \mathrm{H}) \mathrm{ppm}$. Anal. Calcd. For $\mathrm{C}_{28} \mathrm{H}_{19} \mathrm{ClN}_{6} \mathrm{O}_{4}(514.11) \mathrm{C}, 60.64 ; \mathrm{H}, 3.72 ; \mathrm{N}, 16.32$. Found $\mathrm{C}, 60.35 ; \mathrm{H}, 3.51 ; \mathrm{N}, 16.08 \%$.

\subsection{Decarboxylation of 12}

Copper powder $(0.2 \mathrm{~g})$ was added to suspension solution of $\mathbf{1 2}(2.57 \mathrm{~g}, 5 \mathrm{mmol})$ in quinoline $(15 \mathrm{~mL})$. The solution mixture was stirred while being refluxed for $4 \mathrm{~h}$ and cooled. The reaction mixture was poured onto hydrochloric acid $(50 \mathrm{~mL}, 1 \mathrm{~N})$. The solution was extracted with dichloromethane. The organic layer was washed and dried over anhydrous sodium sulfate, then filtered. The solvent was evaporated and the solid that separated was crystallized from dimethylformamide to give a product identical in all respects ( $\mathrm{mp}, \mathrm{mmp}$, and spectral data) with 7 a.

\subsection{Reaction of Compound 6 a with Hydrazonoyl Halides $13 a, b$}

To a hot solution of $6 \mathbf{a}(2.30 \mathrm{~g}, 5 \mathrm{mmol})$ and the appropriate hydrazonoyl halides 13a or 13b $(5 \mathrm{mmol})$ in chloroform $(30 \mathrm{~mL})$ was added triethylamine $(0.7 \mathrm{~mL}, 5 \mathrm{mmol})$. The mixture was refluxed for $18 \mathrm{~h}$. and the solvent was evaporated under reduced pressure. The crude product was collected and crystallized from dimethylformamide to give the products $14 \mathrm{Ba}$ and $14 \mathrm{Bb}$, respectively.

2-(4-Chlorophenyl)-14,15-dimethoxy-2(H)-5-methyl-4-phenylazo-11,12-dihydro-6(H)-1,2,4-triazino[3",2":6',1'] pyrimido[5',4':5,6]pyrido[2,1-a]isoquinoline-1-carbonitrile 14Ba. Mp $280{ }^{\circ} \mathrm{C}$, yield $74 \%$, IR (KBr) $v_{\max } 2191$ $(\mathrm{C} \equiv \mathrm{N}), 3268(\mathrm{NH}) \mathrm{cm}^{-1} .{ }^{1} \mathrm{H}$ NMR $\left(\mathrm{DMSO}_{-} \mathrm{d}_{6}\right) \delta 2.23\left(\mathrm{~s}, 3 \mathrm{H}, \mathrm{CH}_{3}\right), 2.98(\mathrm{~m}, 2 \mathrm{H}$, isoquinoline-4H), $3.78(\mathrm{~s}, 3 \mathrm{H}$, $\left.\mathrm{OCH}_{3}\right), 3.85\left(\mathrm{~s}, 3 \mathrm{H}, \mathrm{OCH}_{3}\right), 3.98(\mathrm{~m}, 1 \mathrm{H}$, isoquinoline- $3 \mathrm{H}), 4.21(\mathrm{~m}, 1 \mathrm{H}$, isoquinoline-3H), $5.24(\mathrm{~s}, 1 \mathrm{H}$, pyridine-4H), $6.77(\mathrm{~s}, 1 \mathrm{H}$, isoquinoline- $8 \mathrm{H}), 7.14-7.70\left(\mathrm{~m}, 9 \mathrm{H}, \mathrm{Ar}{ }^{\prime} \mathrm{H}\right), 7.80(\mathrm{~s}, 1 \mathrm{H}$, isoquinoline-5H), $8.54(\mathrm{~s}, 1 \mathrm{H}$, pyrimidine-2H), $9.03(\mathrm{~s}, 1 \mathrm{H}, \mathrm{NH}) \mathrm{ppm} .{ }^{13} \mathrm{C}$ NMR (DMSO-d $\left.\mathrm{d}_{6}\right) \delta 21.31,28.53,44.90,55.84,55.93,78.91,98.61$, $112.69,118.71,119.69,122.91,122.83,127.63,127.83,128.34,129.61,132.41,133.19,137.63,138.92,141.22$, 142.63, 144.37, 145.26, 145.82, 147.20, 152.01, 152.76, 153.17. MS: m/z 604, 603, 602, 400, 359, 298, 264, 235, 111, 77. Anal. Calcd. For $\mathrm{C}_{33} \mathrm{H}_{27} \mathrm{ClN}_{8} \mathrm{O}_{2}$ (603.07) C, 65.72; H, 4.51; N, 18.58. Found C, 65.35; H, 4.41; N, $18.20 \%$. 
2-(4-Chlorophenyl)-14,15-dimethoxy-2(H)-5-phenyl-4-phenylazo-11,12-dihydro-6(H)-1,2,4-triazino[3",2":6',1'] pyrimido[ $\left[5^{\prime}, 4^{\prime}: 5,6\right]$ pyrido[2,1-a]isoquinoline-1-carbonitrile 14Bb. Mp $290{ }^{\circ} \mathrm{C}$, yield $97 \%$, IR (KBr) $v_{\max } 2191$ $(\mathrm{C} \equiv \mathrm{N}), 3267(\mathrm{NH}) \mathrm{cm}^{-1} .{ }^{1} \mathrm{H}$ NMR $\left(\mathrm{DMSO}_{-} \mathrm{d}_{6}\right) \delta 3.01\left(\mathrm{~m}, 2 \mathrm{H}\right.$, isoquinoline-4H), $3.86\left(\mathrm{~s}, 3 \mathrm{H}, \mathrm{OCH}_{3}\right), 3.91(\mathrm{~s}, 3 \mathrm{H}$, $\left.\mathrm{OCH}_{3}\right), 4.00(\mathrm{~m}, 1 \mathrm{H}$, isoquinoline-3H), $4.26(\mathrm{~m}, 1 \mathrm{H}$, isoquinoline-3H), $5.30(\mathrm{~s}, 1 \mathrm{H}$, pyridine- $4 \mathrm{H}), 6.79(\mathrm{~s}, 1 \mathrm{H}$, isoquinoline-8H), 7.16-7.72 $\left(\mathrm{m}, 14 \mathrm{H}, \mathrm{Ar}^{\prime} \mathrm{H}\right), 7.80(\mathrm{~s}, 1 \mathrm{H}$, isoquinoline- $5 \mathrm{H}), 8.54(\mathrm{~s}, 1 \mathrm{H}$, pyrimidine- $2 \mathrm{H}), 9.03(\mathrm{~s}$, $1 \mathrm{H}, \mathrm{NH}) \mathrm{ppm} .{ }^{13} \mathrm{C}$ NMR $\left(\right.$ DMSO-d $\left._{6}\right) \delta 28.61,45.64,55.73,55.92,78.82,98.12,112.72,118.62,120.73,121.10$, $123.40,127.52,127.68,128.40,128.63,128.82,129.21,129.64,132.32,133.24,135.23,137.68,139.22,143.63$, 144.62, 145.12, 145.25, 145.90, 146.83, 152.21, 153.60, 158.71. MS: m/z 666, 665, 664, 462, 416, 315, 264, 235, 111, 77. Anal. Calcd. For $\mathrm{C}_{38} \mathrm{H}_{29} \mathrm{ClN}_{8} \mathrm{O}_{2}(665.14) \mathrm{C}, 68.61 ; \mathrm{H}, 4.40 ; \mathrm{N}, 16.85$. Found C, 68.30; H, 4.20; N, $16.70 \%$.

2.11 Synthesis of 2-(4-chlorophenyl)-14,15-dimethoxy-2(H)-4-phenylhydrazono-5(4H)-oxo-11,12-dihydro-6(H)1,2,4-triazino[3",2":6', 1']pyrimido[5',4':5,6]pyrido[2,1-a]isoquinoline-1-carbonitrile 16

This compound was prepared using the same method described for the synthesis of $\mathbf{1 4}$ using hydrazonoyl chloride $\mathbf{1 5}$ in place of $\mathbf{1 3 a}, \mathbf{b}$. the compound prepared was crystallized from dimethylformamide, $\mathrm{mp} 190{ }^{\circ} \mathrm{C}$, yield 84\%, IR: (KBr) $v_{\max } 1639(\mathrm{C}=\mathrm{O}), 2190(\mathrm{C} \equiv \mathrm{N}), 3210(\mathrm{NH}), 3310(\mathrm{NH}) \mathrm{cm}^{-1} .{ }^{1} \mathrm{H} \mathrm{NMR}\left(\mathrm{CDCl}_{3}\right) \delta 2.78(\mathrm{~m}$, $2 \mathrm{H}), 3.86\left(\mathrm{~s}, 3 \mathrm{H}, \mathrm{OCH}_{3}\right), 3.91\left(\mathrm{~s}, 3 \mathrm{H}, \mathrm{OCH}_{3}\right), 4.02(\mathrm{~m}, 1 \mathrm{H}), 4.40(\mathrm{~m}, 1 \mathrm{H}), 5.23(\mathrm{~s}, 1 \mathrm{H}$, pyridine-4H), 6.61-8.30 (m, 14H, ArH's, two NH) ppm. Ms: m/z (\%) 606, 605, 400, 315, 236, 235, 111, 77. Anal. Calcd. For $\mathrm{C}_{32} \mathrm{H}_{25} \mathrm{ClN}_{8} \mathrm{O}_{3}$ (605.04) C, 63.52; H, 4.16; N, 18.52. Found C, 63.32; H, 3.92; N, 17.94\%.

\section{Results and Discussion}

The precursors of the title compounds, namely 4-amino-9,10-dimethoxy-2-phenyl-6,7-dihydro-2(H)-pyrido[2,1 -a]isoquinoline-1,3-dicarbonitrile derivatives 4a-c were obtained from 6,7-dimethoxy-3,4-dihydroisoqinoline-1 -acetonitrile 1 (Scheme 1). Thus, treatment of arylidenemalononitrile $\mathbf{2 a - c}$ with $\mathbf{1}$ in boiling acetonitile in the presence of piperidine afforded a single product in each case, as evidenced by TLC analysis. The ${ }^{1} \mathrm{H}$ NMR spectra of all compounds revealed signals near $\delta 4.3\left(\mathrm{~s}, 2 \mathrm{H}, \mathrm{NH}_{2}\right), 4.4(\mathrm{~s}, 1 \mathrm{H},-\mathrm{CHAr}), 6.8-7.4(\mathrm{~m}, \mathrm{Ar}-\mathrm{H})$ in addition to the signals of the dihydroisoquinoline moiety. The IR spectra showed two nitrile absorption bands near $v 2152$ and $2186 \mathrm{~cm}^{-1}$ in addition to the bands of amino group at $v 3354$ and $3471 \mathrm{~cm}^{-1}$. The mass spectrum of each compound 4a-c gave molecular ion peak with high intensity.

Also, in one step the products 4a-c were prepared by refluxing equimolar amounts of isoquinoline-1-acetonitrile, aryl aldehyde and malononitrile in ethanol in the presence of piperidine. The pathway for formation of 4a-c was shown in scheme 1. The reaction started with Michael addition to give $\mathbf{3}$ which upon cyclization led to the formation of 4 .

Reaction of 4a-c each with triethyl orthoformate in acetic anhydride at reflux afforded the ethoxymethyleneamino derivatives $\mathbf{5 a - c}$ in almost quantitative yields (Scheme 2). The structures of the resulting products were confirmed by their elemental analyses, spectral data and their chemical reactions described below. Thus, the IR spectra revealed the absence of the bands of the amino group and the ${ }^{1} \mathrm{H}$ NMR spectra of all products revealed characteristic signals for ethoxy group at $\delta 1.4(\mathrm{t}, \mathrm{J}=7.1 \mathrm{~Hz}, 3 \mathrm{H})$ and at $\delta 4.42(\mathrm{q}, \mathrm{J}=7.1 \mathrm{~Hz}$, $2 \mathrm{H}$ ) as well as a singlet signal at $\delta 8.0$ assignable to a proton of CHOEt group. The mass spectrum of each compound exhibited a molecular ion peak with high intensity.

Treatment of 5a-c with hydrazine hydrate in ethanol at ambient temperature gave the corresponding 4-amino-3-imino-2-aryl-3, 4, 8, 9 -tetrahydro-2(H)-pyrimido [5',4':5,6] pyrido [2,1-a] isoquinoline-1-carbonitrile derivatives 6a-c. The structures proposed for these products were established from their correct elemental analyses and spectroscopic data. Their IR revealed the absence of nitrile absorption frequencies. The ${ }^{1} \mathrm{H}$ NMR spectra for 6a-c showed signals near $\delta 8.64-8.66$ ppm corresponding to the pyrimidine protons, near $\delta 5.86-5.90$ ppm assignable to NH protons and near $\delta 4.75-4.80 \mathrm{ppm}$ assignable to $\mathrm{NH}_{2}$ protons. The ${ }^{13} \mathrm{C}$ NMR spectra of 6 were also compatible with the proposed structure. Further confirmation of the structures of $\mathbf{6 a - c}$ was achieved from their reactions with triethylorthoformate, acetic anhydride and benzoyl chloride. Thus, refluxing compound $\mathbf{6 a}$ in an excess of triethylorthoformate gave product 7a, which was identified on the basis of correct elemental analyses and spectroscopic data as 2-(4-chlorophenyl)-13,14-dimethoxy-10,11-dihydro-2(H)-1,2,4-triazolo $\left[3^{\prime \prime}, 2^{\prime \prime}: 6^{\prime}, 1^{\prime}\right]$ pyrimido $\left[5^{\prime}, 4^{\prime}: 5,6\right]$ pyrido[2,1-a]isoquinoline-1-carbonitrile (Scheme 2). The IR spectrum of $7 \mathbf{a}$ doesn't display absorption bands for the $\mathrm{NH}$ and $\mathrm{NH}_{2}$ groups, which were observed in compound $\mathbf{6 a}$. The ${ }^{1} \mathrm{H}$ NMR spectrum showed two characteristic signals at $\delta 8.32$ and $9.13 \mathrm{ppm}$ assignable to the pyrimidine and the triazole protons respectively. In addition, when 6a-c were refluxed with acetic anhydride or benzoyl chloride in pyridine, it afforded the corresponding 4-methyl and 4-phenyl derivatives 8a-c and 9a-c, respectively (Scheme 2). The structures of 8 and $\mathbf{9}$ were established on both elemental analyses and spectroscopic data. ${ }^{1} \mathrm{H}$ NMR 
spectra of $\mathbf{8}$ and $\mathbf{9}$ showed the absence of the signal of the triazole ring proton at $\delta 9.13 \mathrm{ppm}$, instead, it displayed a singlet signal near $\delta$ 2.45-2.48 ppm assignable to methyl group in compound $\mathbf{8}$.

Compound 6a was refluxed in diethyloxalate and gave a single product 10. Both mass spectrum and elemental analyses were consistent with the formula $\mathrm{C}_{28} \mathrm{H}_{23} \mathrm{ClN}_{6} \mathrm{O}_{4}$. Two possible structures namely $\mathbf{1 0}$ and $\mathbf{1 1}$ can be written for the isolated product formula. The former was assigned to be the isolated product since it gave compound 7a via hydrolysis and decarboxylation of the resulting acid 12 (Scheme 3). The structures of the product $\mathbf{1 0}$ and the corresponding acid $\mathbf{1 2}$ were confirmed by their correct elemental analyses and compatible spectroscopic data (Experimental).

The reaction of 6a with C-acylhydrazonoyl halides 13a,b in refluxing chloroform in the presence of triethylamine afforded, in each case, a single product as evidenced by TLC analysis (Scheme 4). The results of elemental analyses and the mass spectra of the products were consistent with $14 \mathrm{~A}$ and its isomeric structure 14B. The product was assigned structure 14B since the hydrazone structure 14A was excluded depending on their electronic spectra as they revealed a characteristic absorption maxima at $\lambda \max 361(\log \varepsilon 4.43)$ and $\lambda 400(\log \varepsilon$ 4.40) assignable to arylazo chromophore (Burawoy et al., 1952; Yao, 1964; Yao \& Resnick, 1962). The correct structure 14B was further evidenced by spectroscopic data. The IR spectra of 14Ba and 14Bb exhibited an NH absorption band near $v 3268 \mathrm{~cm}^{-1}$ and the ${ }^{1} \mathrm{H}$ NMR spectra showed that the corresponding singlet signal to NH proton at $\delta 9.03 \mathrm{ppm}$ disappeared upon shaking the solution of $14 \mathrm{Ba}$ with deuterium oxide.

Under similar reaction conditions N-phenyl-C-ethoxycarbonylmethanohydrazonoyl chloride $\mathbf{1 5}$ reacted with $\mathbf{6 a}$ and gave compound 16. The IR spectrum of $\mathbf{1 6}$ showed three absorption bands at $v 1639,3210$ and $3310 \mathrm{~cm}^{-1}$ assignable to the amide carbonyl, hydrazone $\mathrm{NH}$ and $\mathrm{NH}$ of triazine groups, respectively.<smiles>COc1cc2c(cc1OC)C(C#N)=NCC2</smiles><smiles>COc1cc2c(cc1OC)C(C([Al])([Al])C([Al])C([N])([N])C#N)=NCC2</smiles><smiles>COc1cc2c(cc1OC)/C(=C(\C#N)C([AlH2])C([AlH2])C#N)NCC2</smiles><smiles>COc1cc2c(cc1OC)C1=C(C)C(C)C(C)C(C)C(C)(C)C(=N)N1CC2</smiles><smiles>COc1cc2c(cc1OC)C1=C(C#N)C([AlH2])C(C#N)=C(N)N1CC2</smiles>

2,4; $\mathrm{Ar}, \mathrm{a}=4-\mathrm{ClC}_{6} \mathrm{H}_{4}, \mathrm{~b}=3,4\left(-\mathrm{O}-\mathrm{CH}_{2}-\mathrm{O}\right) \mathrm{C}_{6} \mathrm{H}_{3}, \mathrm{c}=4-\mathrm{OCH}_{3} \mathrm{C}_{6} \mathrm{H}_{4}$

Scheme 1. Synthesis of pyrido[2,1-a]isoquinoline derivatives 4a-c 


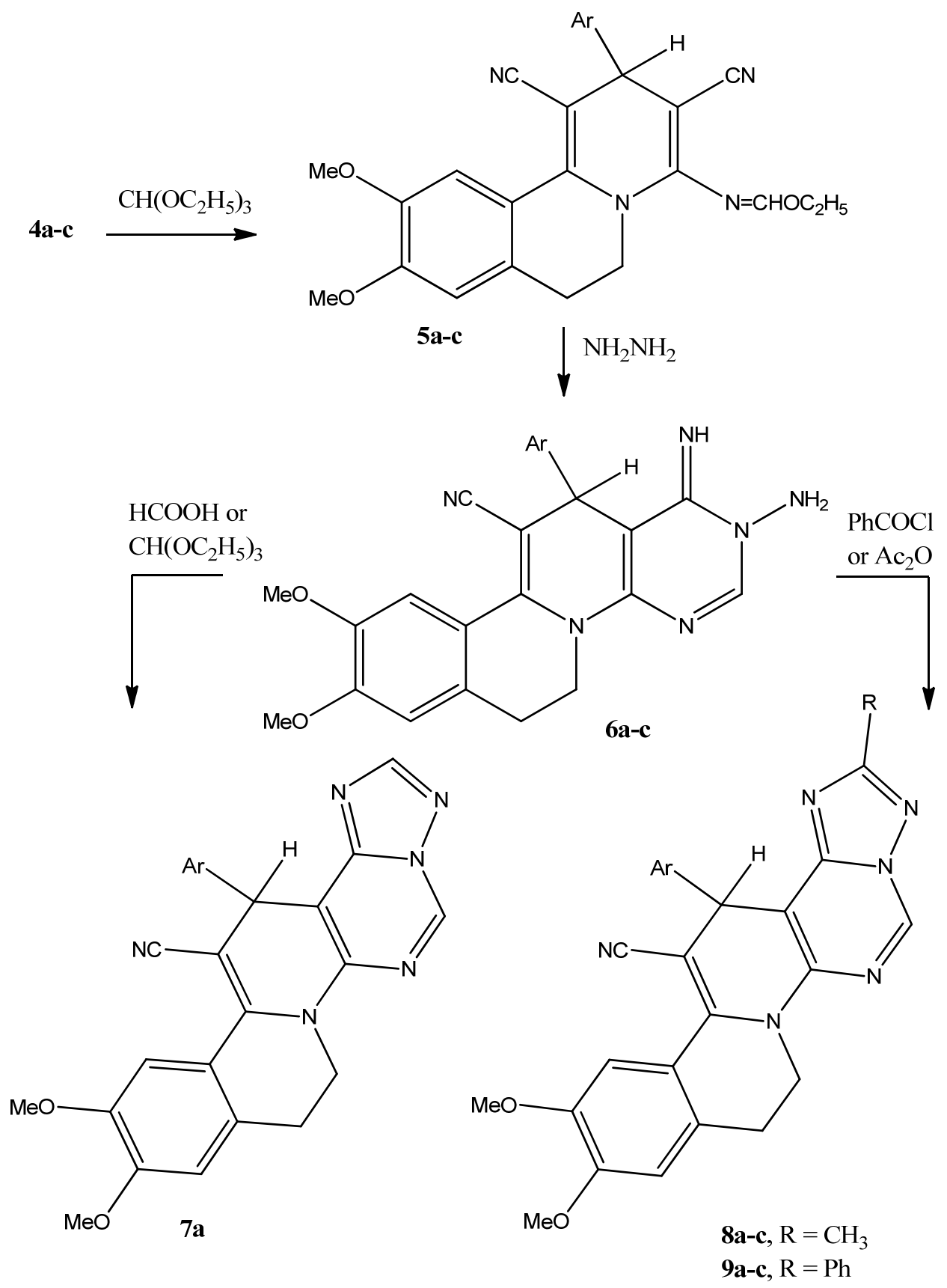

$\mathbf{5 , 7 , 8 , 9 ;} \mathrm{Ar}, \mathrm{a}=4-\mathrm{ClC}_{6} \mathrm{H}_{4}, \mathrm{~b}=3,4\left(-\mathrm{O}-\mathrm{CH}_{2}-\mathrm{O}\right) \mathrm{C}_{6} \mathrm{H}_{3}, \mathrm{c}=4-\mathrm{OCH}_{3} \mathrm{C}_{6} \mathrm{H}_{4}$

Scheme 2. Synthesis of triazolopyrimidopyridoisoquinoline-1-carbonitrile 7, 8 and 9 


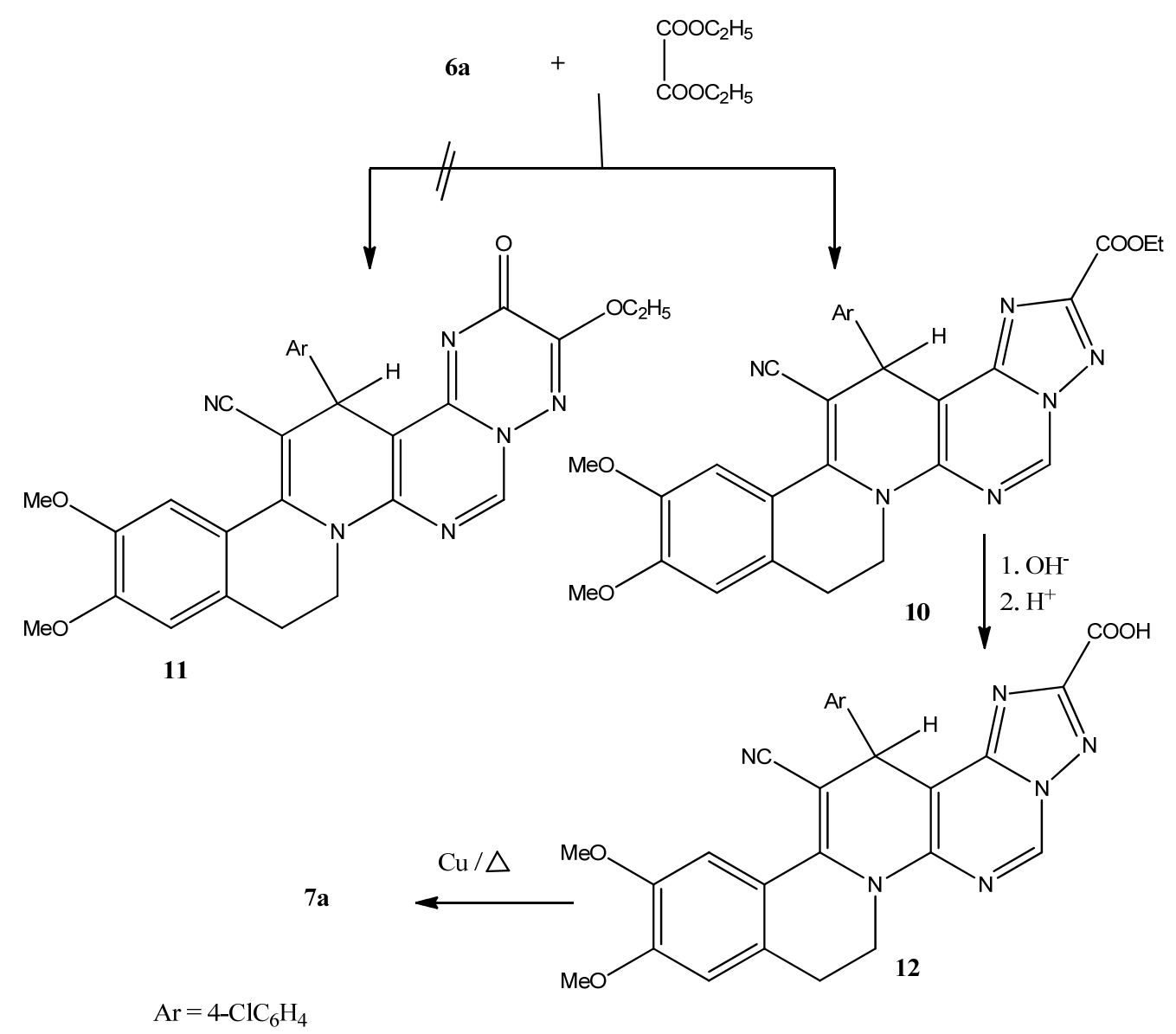

Scheme 3. Synthesis of ethyl triazolopyrimidopyridoisoquinoline-4-carboxylate 10 


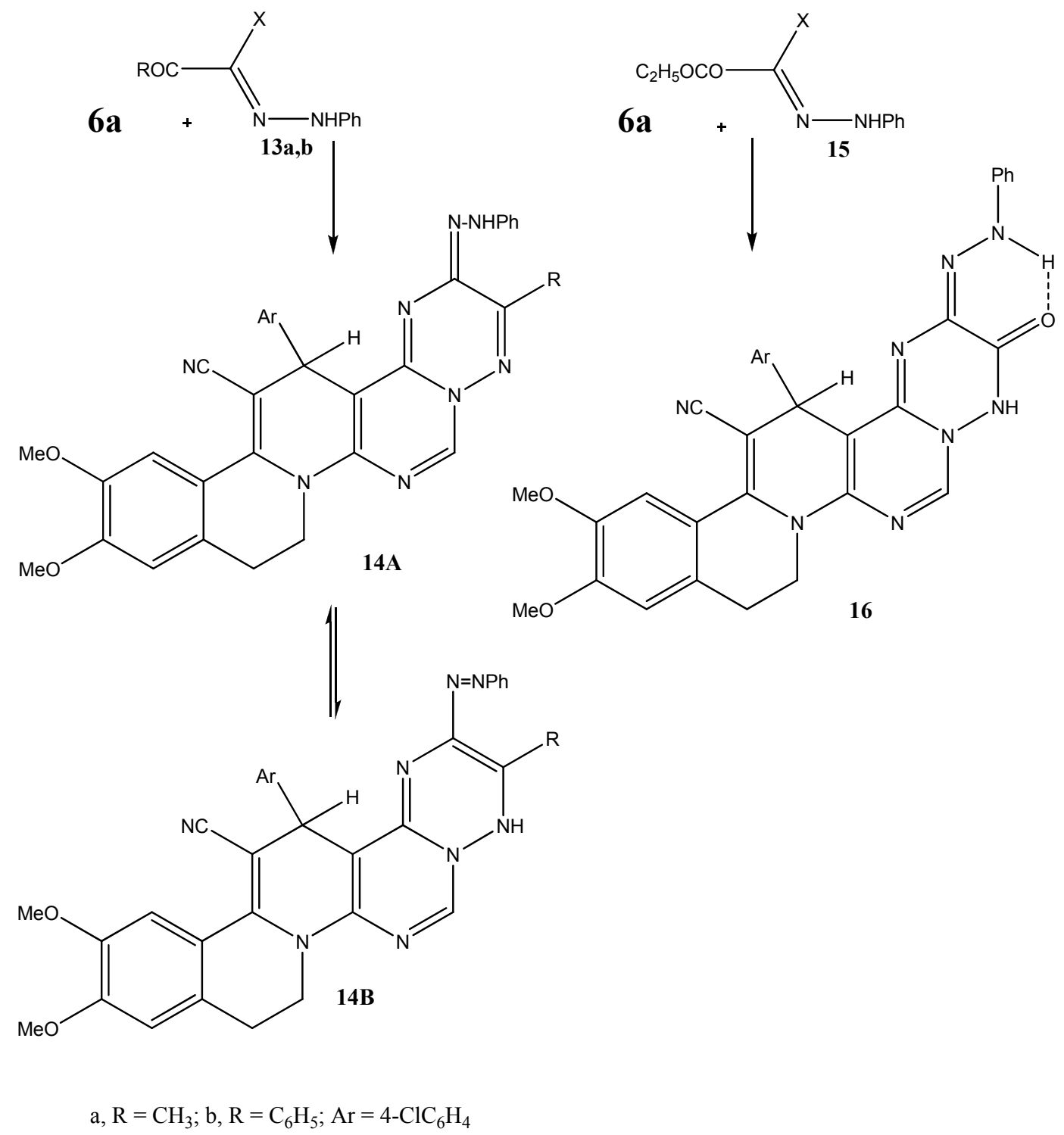

Scheme 4. Synthesis of 4-phenylazo-triazinolopyrimidopyridoisoquinoline-1-carbonitrile 14 and 4-phenylhydrazono-triazinolopyrimidopyridoisoquinoline-1-carbonitrile 16

\section{References}

Abdallah, T. A., Hassaneen, H. M., \& Abdelhade, H. A. (2009). Synthesis of tetra- and penta-heterocyclic compounds incorporated isoquinoline moiety. Heterocycles, 78, 337-345. http://dx.doi.org/10.3987/ COM-08-11481

Barbosa-Filho, J. M., Piuvezam, M. R., Moura, M. D., Silva, M. S., Lima, K. V. B., Leitão da-Cunha, E. V., Fechine, I. M., \& Takemura, O. S. (2006). Anti-infl ammatory activity of alkaloids: A twenty-century. Review. Braz. Pharmaco., 16, 109-139. http://dx.doi.org/10.1590/S0102-695X2006000100020

Boyle, D. L., Kowaluk, E. A., Jarvis, M. F., Lee, C. H., Bhagwat, S. S., Williams, M., \& Firestein, G. S. (2001). Anti-Inflammatory Effects of ABT-702, a Novel Non-Nucleoside Adenosine Kinase Inhibitor, in Rat Adjuvant Arthritis. J. Pharm. Exp. Ther., 296, 495-500.

Buchana, M., Davis, R., Duffy, S., Avery, V., \& Quinn, R. (2009). Antimalarial Benzylisoquinoline Alkaloid from the Rainforest Tree Doryphora sassafras. J. Nat. Prod., 72, 1541-43. http://dx.doi.org/10.1021/ np9002564 
Burawoy, A., Salem, A. G., \& Thompson, A. R. (1952). The nature of the internal hydrogen bond. Part I. Tautomerism of 1-arylazo-2-naphthols. J. Chem. Soc., 4793-98. http://dx.doi.org/10.1039/JR9520004793

Chan, D. C. M., Fu, H., Forsch, R. A., Queener, S. F., \& Rosowsky, A. (2005). Design, Synthesis, and Antifolate Activity of New Analogues of Piritrexim and Other Diaminopyrimidine Dihydrofolate Reductase Inhibitors with $\omega$-Carboxyalkoxy or $\omega$-Carboxy-1-alkynyl Substitution in the Side Chain. J. Med. Chem., 48, 4420-31. http://dx.doi.org/10.1021/jm0581718

Chandra, R., Kaur, J., Talwar, A., \& Ghosh, N. N. (2001). Synthesis and antispasmodic effect of aryl substituted N-carbamoyl/thiocarbamoyl isoquinolines. Arkivoc, VIII, 129-35.

Chen, C. N., Chen, Q., Liu, Y. C., Zhu, X. L., Niu, C. W., Xi, Z., \& Yang, G. F. (2010). Syntheses and herbicidal activity of new triazolopyrimidine-2-sulfonamides as acetohydroxyacid synthase inhibitor. Bioorg. Med. Chem., 18, 4897-4904. http://dx.doi.org/10.1016/j.bmc.2010.06.015

Chung, S. H., Yook, J., Min, B. J., Lee, J. Y., Lee, Y. S., \& Jin, C. (2000). Pharmacological characterization of (10bS)-1,2,3,5,6,10b-hexahydropyrrolo[2,1-a]isoquinoline oxalate (YSL-3S) as a new a2-adrenoceptor antagonist. Arch. Pharm. Res., 23, 360-66.

Elnagdi, M. H., Al-Awdi, N., \& Erian, A. W. (1990). 7.12-Bicyclic 5-6 Systems: Other Four Heteroatoms 2:2 Comprehensive heterocyclic Chem. II, Katrizky, A. R., Rees, C. W., Eserier, Amsterdam, 7, 431-88.

Elwan, N. M., Abdelhadi, H. A., abdallah, T. A., \& Hassaneen, H. M. (1996). Synthesis of $[1,2,4]$ triazolo[3,4-a]isoquinolines and pyrrolo[2,1-a]isoquinolines using $\alpha$-keto hydrazonoyl halides . Tetrahedron, 52, 3451-3456. http://dx.doi.org/10.1016/0040-4020(96)00024-5

Eweiss, N. F., \& Osman, A. (1980). Synthesis of heterocycles. Part II. New routes to acetylthiadiazolines and alkylazothiazoles. J. Heterocycl., Chem., 17, 1713-1717. http://dx.doi.org/10.1002/jhet.5570170814

Hafez, H. N., \& El-Gazzar, A. B. A. (2009). Synthesis and antitumor activity of substituted triazolo[4,3-a]pyrimidin-6-sulfonamide with an incorporated thiazolidinone moiety. Bioorg. Med. Chem. Lett., 19, 4143-47. http://dx.doi.org/10.1016/j.bmcl.2009.05.126

Hafez, H. N., Abbas, N. S., \& El-Gazzar, A. B. A. (2008). Synthesis and evaluation of analgesic, anti-inflammatory and ulcerogenic activities of some triazolo- and 2-pyrazolyl-pyrido [2,3-d]-pyrimidines. Acta. Pharm, 58, 359-78.

Hassaneen, H. M., abdelhadi, H. A., \& Abdallah, T. A. (2001). Novel synthesis of 1,2,4-triazolo[4,3-a]pyrimidin -5-one derivatives. Tetrahedron, 57, 10133-38. http://dx.doi.org/10.1016/S0040-4020(01)01026-2

Kanth, S. R., Reddy, G. V., Kishore, K. H., Rao, P. S., Narsaiah, B., \& Murthy, U. S. N. (2006). Convenient synthesis of novel 4-substitutedamino-5-trifluoromethyl-2,7-disubstituted pyrido[2,3-d] pyrimidines and their antibacterial activity. Eur. J. Med. Chem., 41, 1011-16.

Kashiwada, Y., Aoshima, A., Ikeshiro, Y., Chen, Y. P., Furukawa, H., Itoigawa, M., fujioka, T., Mihashi, K., cosentino, L. M., Morris-Natschke, S. L., \& Lee, K. (2005). Anti-HIV benzylisoquinoline alkaloids and flavonoids from the leaves of Nelumbo nucifera, and structure-activity correlations with related alkaloids. Bioorg. Med. Chem., 13, 443-48.

Knolker, H. C., \& Agarwal, S. (2005).Total synthesis of the antitumor active pyrrolo[2,1-a]isoquinoline alkaloid ( \pm )-crispine A. Tetrahedron Lett., 46, 1173-75. http://dx.doi.org/10.1016/j.tetlet.2004.12.066

Lee, C. H., Jiang, M., Cowar, M., Gfesser, G., Perner, R., Kim, K. H., Gu, Y.G., Williams, M., Jarvis, M. F., Kowaluk, E. A., Stewart, A. O., \& Bhagwat, S. S. S. (2001). Discovery of 4-Amino-5-(3-bromophenyl)-7(6-morpholino-pyridin- 3-yl)pyrido[2,3-d]pyrimidine, an Orally Active, Non-Nucleoside Adenosine Kinase Inhibitor. J. Med. Chem., 44, 2133-38. http://dx.doi.org/10.1021/jm000314x

Markmee, S., Ruchirawat, S., Prachyawarakorn, V., Ingkaninan, K., \& Khorana, N. (2006). Isoquinoline derivatives as potential acetylcholinesterase inhibitors. Bioorg. Med. Chem. Lett., 16, 2170-72. http://dx.doi.org/10.1016/j.bmcl.2006.01.067

Maryanoff, B. E., McComsey, D. f., Constanzo, M. J., Gardick, P. E., Shand, R. P., \& Schneider, C. R. (1984). Pyrroloisoquinoline antidepressants. Potent, enantioselective inhibition of tetrabenazine-induced ptosis and neuronal uptake of norepinephrine, dopamine, and serotonin. J. Med. Chem. 27, 943-46. http://dx.doi.org/10.1021/jm00374a001 
Maryanoff, B. E., McComsey, D. F., Gardocki, J. F., Shank, R. P., Costanzo, M. J., Nortey, S. O., Schneider, C. R., \& Setler, P. E. (1987). Pyrroloisoquinoline antidepressants. 2. In-depth exploration of structure-activity relationships. J. Med. Chem., 30, 1433-54. http://dx.doi.org/10.1021/jm00391a028

Mukherjee, A., Dutta, S., Shanmugavel, M., Mondhe, D. M. Sharma, P. R., Singh, S. K., Saxena, A. K., \& Sanyal U. (2010). 6-Nitro-2-(3-hydroxypropyl)-1H-benz[de]isoquinoline-1,3-dione, a potent antitumor agent, induces cell cycle arrest and apoptosis. J Exp Clin Cancer Res., 29, 175. http://www.jeccr.com/content/29/1/175

Osbond, J. M. (1951). Chemical constitution and amobicidal action. Part I. Synthesis of $\alpha$-tetrahydroisoquinolino- $\omega$-tetrahydro-1-isoquinolylalkanes related to emetine. J. Chem. Soc., 3464-3475. http://dx.doi.org/10.1039/JR9510003464

Phillips, M. A., Gujjar, R., Malmquist, N. A., White, J., El-Mazouni, F., Baldwin, J., \& Rathod P. K. (2008). Triazolopyrimidine-Based Dihydroorotate Dehydrogenase Inhibitors with Potent and Selective Activity against the Malaria Parasite Plasmodium falciparum. J. Med. Chem., 51, 3649-53.http://dx.doi.org/10.1021/jm8001026

Quiroga, J., Insuasty, B., Hormoza, A., Gamerara, D., \& Dominguez, L. J. (1999). Synthesis, characterization and in vitro anthelmintic activity against Nippostrongylus brasiliensis of new 5-aryl-2-phenyl-6,7dihydropyrazolo[1,5-a]pyrimidines. Heterocycl. Chem., 36, 11-13. http://dx.doi.org/10.1002/jhet. 5570360102

Rajagopalan, P. (1984). Synthetic CNS agents. 1,2,3,4,4a,5,10,10a-Octahydro-5,10[1',2']-benzenobenz [g]isoquinoline hydrochloride. A new, highly potent, potential antidepressant. J. Med. Chem., 27, 946-47. http://dx.doi.org/10.1021/jm00374a002

Rawal, R. K., Tripathi, R., Katti, S. B., Pannecouquec, C., \& Clercq, E. D. (2007). Synthesis and evaluation of 2-(2,6-dihalophenyl)-3-pyrimidinyl-1,3-thiazolidin-4-one analogues as anti-HIV-1 agents. Bioorg. Med. Chem., 15, 3134-42. http://dx.doi.org/10.1016/j.bmc.2007.02.044

Shawali, A. S., \& Abdelhamid, A. O. (1976). Reaction of Dimethylphenacylsulfonium Bromide with N-Nitrosoacetarylamides and Reactions of the Products with Nucleophiles. Bull. Chem. Soc. Jpn., 49, 321-324. http://dx.doi.org/10.1246/bcsj.49.321

Shawali, A. S., Eweiss, N. F., Hassaneen, H. M., \& Sami, M. (1975). Synthesis and Rearrangement of Ethyl Aryloxyglyoxalate Arylhydrazones. Bull. Chem. Soc. Jpn., 48, 365-366. http://dx.doi.org/10.1246/ bcsj. 48.365

Sondhi, S. M., Sharma, V. K., Verma, R. P., Singhal, N., Shukla, R., Raghubir, R., \& Dubey, M. P. (1999). Synthesis, Anti-inflammatory and Analgesic Activity Evaluation of Some Mercapto Pyrimidine and Pyrimidobenzimidazole Derivatives. Synthesis, 878-84. http://dx.doi.org/10.1055/s-1999-3472

Vry, J. De., Kuhl, E., Franken-Kunkel, P., \& Echel, G. (2004). Pharmacological characterization of the chronic constriction injury model of neuropathic pain. Eur. J. Pharmacol, 491, 137-48.

Vu, C. B., Shields, P., Peng, B., Kumaravel, G., Jin, X., Phadke, D., Wang, J., Engber, T., Ayyub, E., \& Petter, R. C. (2004). Triamino derivatives of triazolotriazine and triazolopyrimidine as adenosine $\mathrm{A}_{2 \mathrm{a}}$ receptor antagonists. Bioorg. Med. Chem. Lett., 14, 4835-38. http://dx.doi.org/10.1016/j.bmcl.2004.07.048

Yao, H. C. (1964). Azohydrazone Conversion. II. The Coupling of Diazonium Ion with $\beta$-Diketones. J. Org. Chem., 29, 2959-63. http://dx.doi.org/10.1021/jo01033a037

Yao, H. C., \& Resnick, P. (1962). Azo-Hydrazone Conversion. I. The Japp-Klingemann Reaction. J. Am. Chem. Soc., 84, 3514-17. http://dx.doi.org/10.1021/ja00877a018 